\title{
El efecto "Bolonia" en las estrategias y estilos de aprendizaje de los estudiantes de Psicología de la Universidad de La Laguna
}

\section{The "Bolonia" effect on the learning strategies of Psychology students at the University of La Laguna}

\author{
M. Isaura Felipe-Afonso ${ }^{1}$, Andrea Plasencia-Pimentel ${ }^{2}$, Luis Alberto García ${ }^{3}$, José Juan Castro-Sánchez ${ }^{4}$ \\ ${ }^{1}$ Universidad de La Laguna. misaurafa@gmail.com \\ ${ }^{2}$ Universidad de La Laguna. andcpla@gmail.com \\ ${ }^{3}$ Universidad de La Laguna. lagarcia@ull.edu.es \\ ${ }^{4}$ Universidad de Las Palmas de Gran Canaria. josejuan.castro@ulpgc.es
}

Recibido: $24 / 5 / 2021$

Aceptado: 20/10/2021

\section{Copyright $(\mathrm{C}$}

Facultad de CC. de la Educación y Deporte. Universidad de Vigo

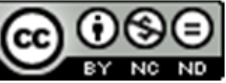

Dirección de contacto: $\mathrm{M}^{\mathrm{a}}$ Isaura Felipe-Afonso Camino Las Camelias, 48 38260 Tejina - La Laguna Santa Cruz de Tenerife

\begin{abstract}
Resumen
El presente trabajo pretende establecer una comparación en el uso de estrategias de aprendizaje entre aquellos estudiantes que realizaron la carrera de Psicología antes de la implantación del Plan Bolonia y aquellos que lo han hecho posteriormente siguiendo el Plan Bolonia. Se ha llevado a cabo un estudio experimental descriptivo con 2.051 estudiantes que cursaban $3^{\circ}$ (Grado) y $4^{\circ}$ (Licenciatura) de Psicología. Se evaluaron las estrategias cognitivas, de control en el estudio, los estilos de aprendizaje y las estrategias motivacionales. Se aplicó una prueba de diferencias de medias t-Student para grupos independientes. Los resultados muestran que los estudiantes con el Plan Bolonia utilizan estrategias cognitivas más reproductivas y colaborativas, así como un estilo de aprendizaje más superficial y teórico. El alumnado No Bolonia se decanta por estrategias cognitivas más productivas, por la búsqueda de alternativas, muestran un estilo más autónomo e inmerso y mayor uso de estrategias motivacionales de concentración y ánimo. Se han encontrado claras diferencias en relación con el grado de autonomía, elaboración y profundización en el estudio y los hábitos motivacionales. Estos datos invitan a una reflexión sobre el impacto de la metodología propuesta por el Plan Bolonia, su implantación y el perfil del estudiante y profesional de la Psicología.
\end{abstract}

\section{Palabras clave}

Bolonia, Estrategias, Aprendizaje, Motivación

\section{Abstract}

The present work aims to establish a comparison in the use of learning strategies between those students who studied psychology before the implementation of the Bologna Plan and those who have done it later following the Bologna Plan. A descriptive experimental study has been carried out with 2.051 students who were in $3^{\text {rd }}$ (Four-years Bachelor) and 4th (Five-years Bachelor) year of Psychology. 
Cognitive strategies, control strategies in the study, learning styles and motivational strategies were evaluated. A t-Student mean difference test was applied for independent groups. The results show that students with the Bologna Plan use more reproductive and collaborative cognitive strategies, as well as a more superficial and theoretical learning style. Non-Bologna students opt for more productive cognitive strategies, search for alternatives, more autonomous and immersive style and greater use of motivational strategies of concentration and encouragement. Clear differences have been found in relation to the degree of autonomy, elaboration and deepening in the study and motivational habits. These data invite a reflection on the impact of the methodology proposed by the Bologna Plan, its implementation and the profile of the student and professional of Psychology.

\section{Key Words}

Bologna, Strategies, Learning, Motivation

\section{INTRODUCCIÓN}

La implantación en las universidades españolas de un nuevo marco educativo común supuso un cambio radical en todos los aspectos relacionados con el proceso de enseñanza-aprendizaje universitario. Esta propuesta planteada desde el Espacio Europeo de Educación Superior (EEES), inicia su transición en el año 2007 mediante un proceso de incorporación progresiva, la cual se instaura en nuestro país en su totalidad en el curso académico 2010-2011.

La aprobación de este nuevo plan académico en España se formaliza a través del Real Decreto 1393/2007, de 29 de octubre, por el que se establece la ordenación de las enseñanzas universitarias oficiales. Esta norma insta a todas las universidades del territorio nacional, que imparten enseñanzas superiores, a "desarrollar la estructura de las enseñanzas universitarias oficiales de acuerdo con las líneas generales emanadas del Espacio Europeo de Educación Superior". Este Real Decreto queda derogado recientemente y se establece un nuevo Real Decreto 822/2021, de 28 de septiembre, por el que se establece la organización de las enseñanzas universitarias y del procedimiento de aseguramiento de su calidad.

En referencia a los principales objetivos que establece el EEES para justificar la implantación a nivel europeo de esta reforma, Díez (2009) destaca los siguientes: homologar los sistemas universitarios, establecer una misma estructura y diseño de las carreras, así como unificar el sistema de créditos y la expedición de los títulos universitarios con validez en todo el territorio europeo. Por otro lado, fomentar la movilidad del alumnado y profesorado universitario entre los países miembros, así como atraer al resto de países del mundo para que elijan realizar su formación superior en Europa. Además, mantener una adecuación constante entre las demandas del mercado laboral y la formación impartida, promoviendo la formación continua y el reciclaje como estilo de vida, es decir, impulsar la actividad formativa mientras se trabaja, dejando atrás la concepción de que la formación y el trabajo son actividades sucesivas e independientes.

En relación con el objeto del presente trabajo, destacamos objetivos como: cambiar y mejorar la metodología de la enseñanza mediante la atención individualizada del estudiante y de su aprendizaje, tal y como plantean Chen et al. (2021) en su estudio, donde destacan el poder de las TICs para lograr un adecuado aprendizaje personalizado 
del alumnado; así como preparar al estudiante para que adopte un papel más activo en su aprendizaje, fomentando su iniciativa por elaborar su propio conocimiento (Quevedo-Blasco y Buela-Casal, 2019). Otra de las metas de la instauración del EEES es apoyar la investigación, para en un futuro lograr una economía a nivel europeo basada en el conocimiento y en el avance científico, y con todo ello, consolidar y certificar la calidad europea a nivel educativo en las instituciones de enseñanza superior (Díez, 2009).

Para llevar a cabo los compromisos asumidos por el plan Bolonia, los países que comenzaron con la reforma se apoyaron principalmente en el desarrollo de tres herramientas (European Commission, Education Audiovisual and Culture Executive Agency, 2020, pp.39-43):

- Suplemento Europeo al Título: se trata de un documento estandarizado que describe la naturaleza y estado de los estudios realizados por la persona titular del diploma original. Esta herramienta busca la mejora de la transparencia y el reconocimiento de las cualificaciones a nivel europeo.

- Marco de referencia Nacional de Cualificaciones: permite la fiabilidad y equivalencia de las calificaciones, tanto dentro de un mismo país como entre países, con el objetivo de mostrar las diferencias encontradas entre las calificaciones.

- Sistema Europeo de Transferencia y Acumulación de Créditos: se trata de un sistema de reconocimiento del aprendizaje obtenido en instituciones del propio país o fuera del mismo, traduciéndolo en créditos compatibles. El sistema de ECTS permite reconocer todas las formas de aprendizaje dentro de la estructura formal de la Educación Superior.

Junto con estas nuevas herramientas, también se procedió a la eliminación de las licenciaturas y diplomaturas para implementar una nueva estructura de titulaciones basada en dos niveles: Grado (Primer Ciclo) y Postgrado (Segundo Ciclo), y este último dividido en Máster y Doctorado (Bolívar, 2007; Cazorla, 2011).

Según Bolívar (2007), y en consonancia con los objetivos señalados anteriormente, la clave fundamental y la principal motivación de la propuesta de dicha reforma, no parece focalizarse en la adaptación de los planes de estudio a las nuevas estructuras sino en un cambio de la "cultura de la enseñanza universitaria" enfocada en el desarrollo de competencias generales y específicas (Dirección General de Educación y Cultura, 2002; Corominas, 2001; Perrenoud, 2001; Rué, 2007), centrada en el aprendizaje de los estudiantes y en un rediseño de la práctica del profesorado.

Debido al gran impacto que supuso esta adaptación en todos los niveles de la educación universitaria española, consideramos imperativo estudiar y conocer el efecto que, una vez asumida por completo esta transición, ha supuesto en la forma de aprender de los estudiantes universitarios (Amber y Martínez-Valdivia, 2018). Otro estudio desarrollado en esta línea ha aportado interesantes resultados sobre la satisfacción del profesorado sobre la incorporación al EEES (Quevedo-Blasco y Buela-Casal, 2017).

Tal y como se ha señalado anteriormente, uno de los fundamentos que han avalado este cambio, ha sido la mejora de la metodología de enseñanza-aprendizaje universitario con la implantación de nuevas estrategias. Por ello, con este estudio pretendemos comprobar si después de 10 años desde su instauración en nuestro país, se ha logrado alcanzar dicho fin y si la incorporación al EEES ha mejorado realmente las estrategias y estilos de aprendizaje de nuestro alumnado. Pretendemos, además, aportar un valor 
añadido a los actuales métodos de analítica del aprendizaje (Learning Analytics) del alumnado, basados en los datos arrojados producto de la interacción de los estudiantes universitarios con los contenidos académicos de las aulas virtuales y la formación online (Guzmán-Valenzuela et al., 2021).

G. López et al. (2010) afirman que la implantación del plan Bolonia persigue el cambio de la enseñanza magistral y directiva a un aprendizaje primordialmente práctico y autónomo que logre disminuir la dependencia del alumnado con el profesorado durante su aprendizaje. Este fin se pretende alcanzar mediante el desarrollo y fomento en los estudiantes de estrategias de aprendizaje basadas en la organización, comprensión y síntesis del contenido, el trabajo colaborativo y el fomento de la creación de informes, tareas, trabajos y exposiciones orales de los productos académicos, como herramientas de trabajo habitual del alumnado. Según las aportaciones de Gutierrez-Braojos et al. (2009), el hecho de que los alumnos y alumnas aprendan de forma conjunta, es decir, cooperando con sus iguales, asumiendo su parte de responsabilidad individual y aceptando la responsabilidad de grupo correspondiente, parece facilitar la asimilación de estrategias de autorregulación.

Por otro lado, M.I. López et al. (2016) señalan que las recomendaciones planteadas por el EEES incluyen un importante aumento del uso de las TIC's a través de las plataformas virtuales creadas por las instituciones para el alumnado. Y con ello también, un considerable incremento de la carga de trabajo de los universitarios, así como la aplicación de metodologías didácticas novedosas con el objetivo de incrementar la calidad del proceso de enseñanza-aprendizaje. Sin embargo, según este autor, se plantearon todas estas modificaciones sin disponer de los recursos necesarios tanto humanos como materiales para poder llevarlo a cabo.

Según Correa (2019), la llegada del plan Bolonia y del Espacio Europeo de Investigación a las universidades de América Latina, supusieron la simplificación de la investigación en las Ciencias Sociales y Humanidades, consiguiendo con ello aumentar el grado de titulación del alumnado universitario.

Todos estos cambios y modificaciones en la estructura y forma de la enseñanza superior, han promovido muchas reflexiones desde toda la comunidad educativa y social en nuestro país. Con el presente trabajo se pretende estudiar el impacto que tuvo y sigue teniendo el plan Bolonia en el proceso de aprendizaje de los estudiantes universitarios. Concretamente analizamos los efectos en el estilo de aprendizaje, en el uso de estrategias cognitivas y de control en el estudio, así como en los hábitos motivacionales para el estudio de los estudiantes del Grado de Psicología de la Universidad de La Laguna (ULL), en comparación con los estudiantes de la Licenciatura de Psicología del plan de estudios anterior a la implantación de Bolonia de la misma Facultad.

Particularmente, en referencia a los estilos de aprendizaje del alumnado universitario, Brosig et al. (2021) indican en su reciente publicación que para alcanzar un aprendizaje significativo es necesario realizar previamente un análisis del estilo de aprendizaje del alumnado. Con esta información el profesorado podrá ajustar su metodología didáctica al estilo de aprendizaje del alumnado y poder intervenir con mayor éxito en las carencias de los estudiantes (Soflano et al., 2015).

En cuanto a la motivación por el estudio, un estudio realizado sobre el proceso de enseñanza-aprendizaje en las universidades populares de España, sostiene que el uso de TIC's genera una mayor motivación del alumnado por el aprendizaje. Por otro lado, 
asegura que la metodología de trabajo colaborativo tanto entre alumnos y alumnas como entre alumnado-profesorado, se establece como la base didáctica del proceso enseñanzaaprendizaje para la consecución de las metas propuestas en cada curso académico. Por último, destaca sobre todo, que la metodología didáctica principal se centra en la enseñanza personalizada, la cual se diseña según las necesidades que presente cada alumno y alumna; una metodología activa, donde el estudiante es el protagonista y a su vez responsable de su propio proceso de aprendizaje (J.A. López et al., 2009).

Una reciente investigación indica que el uso adecuado de estrategias de aprendizaje por parte del alumnado contribuye significativamente a una apropiada adquisición de contenidos académicos, a la memorización, codificación y capacidad de discriminar eficazmente las fuentes de información más acertadas (Trelles et al., 2018). Según Teijeiro y Requies (2018), las estrategias de aprendizaje se utilizan para adquirir conocimientos de forma intencionada hacia el logro de una meta concreta, por lo que el estudiante organiza los recursos y capacidades disponibles para su consecución.

Se ha demostrado que la habilidad de autocontrol es una de las características esenciales para lograr convertirse en un estudiante autorregulado (Zimmerman, 2000; Zimmerman y Dibenedetto, 2008; Zimmerman y Martinez-Pons, 1988). En referencia a las estrategias de control en el estudio, Hendrie y Bastacini (2020) en su investigación sobre el proceso de autorregulación en el estudio, señalan que una mayor flexibilidad a la hora de disponer de sus propios criterios para elegir las estrategias de aprendizaje más adecuadas según la materia de estudio o tarea concreta promueve el logro de mejores resultados académicos. Por lo tanto, según estas aportaciones, el fomento de la autonomía en los estudiantes desde el proceso de enseñanza-aprendizaje facilita la consecución de los objetivos propuestos (Juárez et al., 2016).

Estas aportaciones coinciden con los hallazgos aportados por Monereo (2020), quien sostiene que para que el alumnado consiga ser autónomo y logre disminuir su grado de dependencia con el profesorado, se requiere que aplique un aprendizaje estratégico a lo largo de su etapa universitaria. Este aprendizaje estratégico requiere que el alumnado tome decisiones sobre cómo y cuándo aprender según el tipo de contenido y tarea a la que se enfrente en cada momento. Otras investigaciones afirman que existe una notable correspondencia entre la forma de aprender de los estudiantes y el resultado académico alcanzado (Esguerra y Guerrero, 2010).

Las conclusiones proporcionadas por estos estudios en cuanto a la forma de aprendizaje del alumnado se muestran coherentes con los objetivos y recomendaciones marcadas por el EEES. No obstante, nos planteamos como hipótesis si se ha conseguido llevar a la práctica las teorías propuestas por el plan Bolonia en lo referente al proceso de enseñanza-aprendizaje. Si realmente se ha logrado una mejora en las estrategias y estilos de aprendizaje y hábitos motivacionales del alumnado universitario perteneciente al Grado en Psicología, gracias a la incorporación al EEES; o si por el contrario esta modificación ha supuesto una pérdida de competencias y estrategias de aprendizaje óptimas que los planes de estudio anteriores aportaban eficazmente al alumnado de Psicología.

Para la realización de este estudio se eligió la batería de pruebas HERGAR, la cual cuenta con un sólido modelo teórico desarrollado en el libro Psicología y enseñanza del estudio (Hernández y García, 1991). Además, estas pruebas se han aplicado en otros trabajos anteriores como por ejemplo Martín et al. (2007, 2008), entre otras publicaciones. 


\section{MÉTODO}

\subsection{Muestra}

La presente investigación se ha realizado con la participación de 2.051 alumnos y alumnas pertenecientes a grupos tanto de horario de mañana como de tarde de la Universidad de La Laguna. En un primer momento se recogieron los datos del alumnado que cursaba $4^{\circ}$ curso de la Licenciatura de Psicología, desde la promoción de 2003/2004 hasta el curso académico 2008/2009. Posteriormente, una vez que el plan Bolonia estuvo instaurado por completo, se aplicaron las pruebas a los estudiantes del $3^{\circ}$ curso del Grado de Psicología, desde la promoción de 2013/2014 hasta el curso académico 2020/2021. La distribución de los participantes según el plan académico que les ha correspondido ha sido con el plan Bolonia el $67,8 \%$ y el $32,3 \%$ con el plan anterior, tal y como se muestra en la siguiente tabla:

\begin{tabular}{ccc}
\hline & $\mathrm{N}$ & $\%$ \\
\hline Bolonia & 1.390 & 67,8 \\
\hline No Bolonia & 661 & 32,2 \\
\hline
\end{tabular}

Tabla 1. Valores y porcentajes de la muestra según el plan académico

Los datos sociodemográficos que hemos considerado relevantes para la realización de este estudio se exponen a continuación:

\begin{tabular}{ccc}
\hline & $\mathrm{N}$ & $\%$ \\
\hline Mujeres & 1.356 & 66,1 \\
\hline Hombres & 335 & 16,3 \\
\hline No informan & 360 & 17,6 \\
\hline
\end{tabular}

Tabla 2. Valores y porcentajes de la muestra según su sexo

Tal y como se puede observar en la Tabla 2, la muestra está formada mayoritariamente por mujeres con un $66,1 \%$ de representación en la muestra, los hombres con un $16,3 \%$ de participación y un $17,6 \%$ de los alumnos y alumnas decidieron no informar sobre su género.

\begin{tabular}{ccc}
\hline & $\mathrm{N}$ & $\%$ \\
\hline $18-19$ & 63 & 3,1 \\
\hline 20 & 690 & 33,6 \\
\hline 21 & 379 & 18,5 \\
\hline 22 & 224 & 10,9 \\
\hline 23 & 123 & 6 \\
\hline $24-25$ & 153 & 7,4 \\
\hline $26-30$ & 85 & 4,2 \\
\hline $31-61$ & 60 & 2,9 \\
\hline No informan & 274 & 13,4
\end{tabular}

Tabla 3. Valores y porcentajes de la muestra según su edad 
En cuanto a la edad del alumnado participante en el estudio, encontramos en la Tabla 3 que la mayor parte de la muestra se encuentra representada por jóvenes de entre $20 \mathrm{y}$ 22 años, agrupando así a más del 60\% del total de estudiantes en este rango de edad.

Por último y en referencia a los porcentajes sobre el sexo y la edad con la etiqueta "No informan", estos agrupan a aquellos participantes que decidieron no informar de estas variables. Consideramos necesario no obligar a los estudiantes a aportar esta información para no condicionar su respuesta o la realización de las pruebas a la inclusión de datos sociodemográficos que el estudiante considerara íntimos. Afortunadamente, el porcentaje no informado no perjudicó el estudio.

\subsection{Instrumentos}

Para llevar a cabo esta investigación se han utilizado cuatro de las cinco pruebas que conforman la batería de cuestionarios de HERGAR (Hernández y García, 1991) y se describen a continuación:

- Cuestionario sobre Estrategias Cognitivas de Aprendizaje (ECA) (Hernández y García, 1991): este cuestionario cuenta con un total de 44 ítems agrupados en 11 factores: estructuración, elaboración, esencialización, aprendizaje receptivo, comprensión elaborada, aprendizaje mecánico-superficial, voz alta, nemotecnia, aprendizaje maximalista, aprendizaje repetitivo y aprendizaje colaborativo y oral. En relación a la consistencia interna de este instrumento, cuenta con un coeficiente de fiabilidad $\alpha$ de Cronbach de, 965 .

- Cuestionario sobre Estrategias de Control en el Estudio (ECE) (Hernández y García, 1991): este cuestionario cuenta con un total de 17 ítems agrupados en 3 factores: revisión, planificación y búsqueda de alternativas. Esta prueba cuenta con un coeficiente de fiabilidad $\alpha$ de Cronbach de ,867.

- Cuestionario sobre Estilos de Aprendizaje (ESTAP) (Hernández y García, 1991): esta prueba está compuesta por un total de 100 ítems agrupados en 13 factores: estilo productivo, estilo autorregulado, estilo estratégico demandas, estilo teórico, estilo básico, estilo convergente, estilo superficial, estilo divergente, estilo práctico en el estudio, estilo reproductivo, estilo inmerso, estilo atomista y estilo preferencia trabajo personal. El coeficiente de fiabilidad $\alpha$ de Cronbach es ,731 para este instrumento.

- Cuestionario sobre Hábitos y Estrategias Motivacionales para el Estudio (HEME) (Hernández y García, 1991): esta prueba está compuesta por un total de 44 ítems agrupados en 11 factores: hábitos de interés y concentración, superación de las dificultades emocionales, actividad y egoimplicación, valoración logros, sentido funcional, interconexión y ampliación, aproximación gradual, anticipación facilitadora, descarga ante tensión, descanso y variación, relajación y ánimo. Para esta prueba el coeficiente de fiabilidad $\alpha$ de Cronbach es ,886.

En todas las pruebas, se le solicita al estudiante que indique su nivel de acuerdo con cada ítem, siendo la forma de respuesta en todos los cuestionarios, de tipo Likert (1-5). Además, el alumnado fue informado de que los datos obtenidos serían tratados con absoluta confidencialidad y solo se manejarían resultados globales. De esta forma, se les alentó para que contestasen con total sinceridad y con ello colaborasen con la presente investigación. 


\subsection{Procedimiento}

Inicialmente, la recogida de los datos del alumnado de Licenciatura en Psicología se llevó a cabo manualmente. Es decir, se entregaron los cuestionarios (Batería de Cuestionario HERGAR) en formato papel a los estudiantes de $4^{\circ}$ curso matriculados en la asignatura troncal "Evaluación e Intervención Psicoeducativa" y se introdujeron los datos obtenidos en el programa estadístico "Statistical Package for the Social Sciences" (SPSS). En esta época los formularios de Google no estaban aún disponibles como herramienta de investigación.

Posteriormente, a partir de 2014, se realizó esta recogida de información mediante un cuestionario online utilizando la plataforma de formularios de Google, incorporando en este los instrumentos desarrollados anteriormente. De esta forma, se consiguió hacerlo llegar a todos los alumnos y alumnas a través de un enlace online que les permitía acceder a los cuestionarios. El acceso a las pruebas se encontraba disponible en el campus virtual de la Universidad de La Laguna para los estudiantes de $3^{\circ}$ curso matriculados en la asignatura troncal "Evaluación e Intervención Psicoeducativa", correspondiente a la titulación del Grado en Psicología de la Universidad de La Laguna.

Tanto el profesorado encargado de impartir la asignatura de "Evaluación e Intervención Psicoeducativa" como el equipo de investigación encargado de gestionar los datos y realizar los análisis pertinentes, pertenecían a la Universidad de La Laguna desde el inicio de la aplicación de las pruebas. Por ese motivo la muestra está compuesta por alumnado matriculado en dicha asignatura impartida en el $4^{\circ}$ curso de Licenciatura y el $3^{\circ}$ curso del Grado y perteneciente a la Facultad de Psicología de la ULL.

El alumnado realizó las pruebas siendo consciente de que formaba parte de una actividad para dicha asignatura. Prácticamente el 100\% de los alumnos las realizaron. Para controlar la correcta participación de los estudiantes matriculados en la asignatura, se solicitó el Documento Nacional de Identidad como medio identificativo para conocer quién accedía a la actividad, pero nunca el nombre ni los apellidos. No obstante, se les informó presencialmente que sus datos serían tratados por un equipo de investigación ajeno al profesorado evaluador de la asignatura. El profesorado solo contaría con la lista de participantes en la actividad, cedida por el equipo encargado de la gestión de datos.

El equipo de investigación recomendó a los estudiantes contar con tiempo suficiente y un lugar apropiado para la realización de las pruebas con el objetivo de no perjudicar la fiabilidad de los resultados, teniendo en cuenta el factor cansancio tanto para el formato papel como para el online.

Confirmamos que este estudio se ha llevado a cabo con el consentimiento plenamente informado del alumnado y con la aprobación formal del comité de investigación y ética de la Universidad de La Laguna en referencia al protocolo aplicado.

\section{ANÁLISIS DE LOS DATOS}

Los datos han sido analizados a través del programa estadístico "Statistical Package for the Social Sciences" (SPSS), versión 25.

Con el objetivo de poder contrastar las hipótesis que se han planteado para este estudio, se han creado dos grupos de participantes según el plan de estudios que les 
correspondiera en el momento de realizar sus estudios universitarios. En este caso, la Licenciatura de Psicología antes de 2007 se llevó a cabo con la Ley Orgánica de Universidades (LOU) y para el Grado de Psicología, a partir de 2007, los alumnos y alumnas obtuvieron su titulación mediante el plan Bolonia (Ley Orgánica 4/2007, de 12 de abril, la cual modifica la Ley Orgánica 6/2001, de 21 de diciembre, de Universidades). Es por ello, que los grupos creados para proceder al análisis de los datos se clasifican entre Bolonia $(\mathrm{N}=1.390)$ y No Bolonia $(\mathrm{N}=661)$, correspondiéndose con la denominación de ambos grupos.

Una vez clasificado el alumnado según su plan de estudios, se aplicó una prueba de diferencias de medias t-Student para grupos independientes sobre la variable Plan Académico. Con este análisis de datos se pretende hallar aquellas estrategias cognitivas de aprendizaje, estrategias de control del estudio, estrategias motivacionales y estilos de aprendizaje más frecuentes en cada uno de los grupos que se estudian y comprobar la existencia de diferencias significativas entre ambos.

\subsection{Resultados}

En este apartado se presentarán los resultados obtenidos una vez aplicada la prueba de diferencias de medias t-Student para grupos independientes sobre la variable Plan Académico.

Como puede observarse en las siguientes tablas, se han encontrado diferencias significativas en la prueba de muestras independientes de las medias de los grupos Bolonia y No Bolonia para los factores de la batería de cuestionarios HERGAR. Dada la magnitud de resultados significativos obtenidos en este análisis y siendo más exigentes con nuestros hallazgos, hemos decidido destacar e interpretar principalmente aquellos datos arrojados con $\mathrm{p}<, 000$.

Profundizando en la Tabla 4, encontramos que en comparación con el alumnado No Bolonia, los alumnos y alumnas del grupo Bolonia utilizan estrategias cognitivas más relacionadas con el aprendizaje mecánico-superficial $(\mathrm{t}=8,41 ; \mathrm{p}<, 000)$, el aprendizaje en voz alta $(\mathrm{t}=5,79 ; \mathrm{p}<, 000)$, la mnemotécnica $(\mathrm{t}=3,71 ; \mathrm{p}<, 000)$, repetitivo $(\mathrm{t}=5,06$; $\mathrm{p}<, 000)$ y colaborativo y oral $(\mathrm{t}=5,42 ; \mathrm{p}<, 000)$. Por su parte, el grupo No Bolonia utiliza más estrategias de esencialización $(\mathrm{t}=-6,54 ; \mathrm{p}<, 000)$ y de comprensión elaborada $(\mathrm{t}=-5,88 ; \mathrm{p}<, 000)$, en comparación con el grupo Bolonia.

Tal y como se puede observar en la Tabla 5, en referencia a las estrategias de control del estudio, encontramos que el grupo de estudiantes No Bolonia utiliza en mayor medida la búsqueda de alternativas $(\mathrm{t}=-3,85 ; \mathrm{p}<, 000)$, como estrategia de control del estudio en comparación con el grupo Bolonia.

Por otro lado, la Tabla 6 muestra cómo los alumnos y alumnas pertenecientes al grupo Bolonia poseen un estilo de aprendizaje teórico $(\mathrm{t}=4,12 ; \mathrm{p}<.000)$ y superficial $(\mathrm{t}=$ $7,69 ; \mathrm{p}<, 000)$. En el caso de los estudiantes del grupo No Bolonia destacan los estilos de aprendizaje autorregulado $(\mathrm{t}=-3,53 ; \mathrm{p}<, 000)$ e inmerso $(\mathrm{t}=-4,87 ; \mathrm{p}<, 000)$. 


\begin{tabular}{ccccccc}
\hline & $\begin{array}{c}\mathrm{x} \overline{1} \\
\text { Bolonia }\end{array}$ & $\mathrm{DT}$ & $\begin{array}{c}\mathrm{x} \overline{2} \\
\text { NoBolonia }\end{array}$ & $\mathrm{DT}$ & $\mathrm{t}(\mathrm{gl})$ & $\mathrm{P}$ \\
\hline Estructuración & 3,43 &, 750 & 3,48 &, 739 & $-1,47(1.310)$ &, 141 \\
\hline Elaboración & 3,56 &, 753 & 3,60 &, 755 & $-1,17(1.282,41)$ &, 242 \\
\hline Esencialización & 3,73 &, 603 & 3,91 &, 559 & $-6,54(1.380,15)$ &, $000^{*}$ \\
\hline Receptivo & 2,25 &, 768 & 2,18 &, 779 & $1,89(1.260,89)$ &, 059 \\
\hline $\begin{array}{c}\text { Comprensión } \\
\text { Elaborada }\end{array}$ & 2,48 &, 500 & 2,62 &, 504 & $-5,88(1.279,69)$ &, $000^{*}$ \\
\hline $\begin{array}{c}\text { Mecánico - } \\
\text { Superficial }\end{array}$ & 2,35 &, 710 & 2,09 &, 636 & $8,41(1.431,64)$ &, $000^{*}$ \\
\hline Voz Alta & 3,41 & 1,046 & 3,13 & 1,018 & $5,79(1.329,61)$ &, $000^{*}$ \\
\hline Mnemotécnica & 3,57 & 1,033 & 3,39 & 1,018 & $3,71(1.314,61)$ &, $000^{*}$ \\
\hline Maximalista & 2,38 &, 630 & 2,32 &, 534 & $2,30(1.488,66)$ &, 022 \\
\hline Repetitivo & 2,94 &, 919 & 2,72 &, 936 & $5,06(1.276,06)$ &, $000^{*}$ \\
\hline $\begin{array}{c}\text { Colaborativo y } \\
\text { Oral }\end{array}$ & 3,00 & 1,008 & 2,76 &, 929 & $5,42(1.395,32)$ &, $000^{*}$ \\
\hline
\end{tabular}

$* \mathrm{p}<, 000$

Tabla 4. Diferencias de medias t-Student para muestras independientes (ECA)

\begin{tabular}{ccccccc}
\hline & $\begin{array}{c}\mathrm{x} \overline{1} \\
\text { Bolonia }\end{array}$ & DT & $\begin{array}{c}\mathrm{x} \overline{2} \\
\text { NoBolonia }\end{array}$ & DT & $\mathrm{t}(\mathrm{gl})$ & $\mathrm{P}$ \\
\hline Revisión & 3,88 &, 689 & 3,93 &, 566 & $-1,72(1.546,04)$ &, 086 \\
\hline Planificación & 3,90 &, 802 & 3,94 &, 676 & $-1,21(1.511,44)$ &, 228 \\
\hline $\begin{array}{c}\text { Búsqueda de } \\
\text { Alternativas }\end{array}$ & 3,40 &, 573 & 3,50 &, 520 & $-3,85(1.417,39)$ &, $000^{*}$ \\
\hline
\end{tabular}

$* \mathrm{p}<, 000$

Tabla 5. Diferencias de medias t-Student para muestras independientes (ECE)

\begin{tabular}{ccccccc}
\hline & $\begin{array}{c}\mathrm{x} \overline{1} \\
\text { Bolonia }\end{array}$ & $\mathrm{DT}$ & $\begin{array}{c}\mathrm{x} \overline{2} \\
\text { NoBolonia }\end{array}$ & $\mathrm{DT}$ & $\mathrm{t}(\mathrm{gl})$ & $\mathrm{P}$ \\
\hline Productivo & 3,24 &, 640 & 3,30 &, 629 & $-1,88(1.181,37)$ &, 061 \\
\hline Autorregulado & 3,74 &, 577 & 3,83 &, 537 & $-3,53(1.285,64)$ &, $000^{*}$ \\
\hline $\begin{array}{c}\text { Estratégico } \\
\text { Demandas }\end{array}$ & 3,35 &, 855 & 3,30 &, 851 & $1,11(1.282,14)$ &, 266 \\
\hline Teórico & 3,41 &, 740 & 3,27 &, 665 & $4,12(1.420,50)$ &, $000^{*}$ \\
\hline Básico & 2,28 &, 675 & 2,36 &, 670 & $-2,73(1.302,10)$ &, 006 \\
\hline Convergente & 3,05 &, 817 & 3,17 &, 795 & $-3,24(1.308,33)$ &, 001 \\
\hline Superficial & 2,67 &, 778 & 2,40 &, 736 & $7,69(1.360,53)$ &, $000^{*}$ \\
\hline Divergente & 3,53 &, 697 & 3,60 &, 664 & $-2,09(1.315,13)$ &, 037 \\
\hline $\begin{array}{c}\text { Práctico en el } \\
\text { Estudio }\end{array}$ & 2,98 &, 517 & 3,00 &, 494 & $-0,70(1.312,20)$ &, 486 \\
\hline Reproductivo & 2,43 &, 730 & 2,49 &, 769 & $-1,82(1.239,36)$ &, 069 \\
\hline Inmerso & 2,39 &, 769 & 2,56 &, 746 & $-4,87(1.311,33)$ &, $000^{*}$ \\
\hline Atomista & 2,45 &, 725 & 2,38 &, 682 & $2,00(1.363,70)$ &, 046 \\
\hline $\begin{array}{c}\text { Preferencia } \\
\text { Trabajo }\end{array}$ & 3,42 &, 778 & 3,43 &, 720 & $-0,31(1.375,81)$ &, 757 \\
Personal & & & & & & \\
\hline * p $<.000$ & & & & & & \\
\hline
\end{tabular}

Tabla 6. Diferencias de medias t-Student para muestras independientes (ESTAP) 
En referencia a los hábitos y estrategias motivacionales llevadas a cabo por los participantes de este estudio, encontramos en la Tabla 7 cómo el grupo No Bolonia muestra, en mayor medida, hábitos de interés y concentración $(\mathrm{t}=-3,78 ; \mathrm{p}<, 000)$, descarga ante tensión $(\mathrm{t}=-5,76 ; \mathrm{p}<, 000)$ y relajación y ánimo $(\mathrm{t}=-4,34 ; \mathrm{p}<, 000)$ en comparación con el grupo Bolonia.

\begin{tabular}{|c|c|c|c|c|c|c|}
\hline & $\begin{array}{c}\text { x } \overline{1} \\
\text { Bolonia }\end{array}$ & DT & $\begin{array}{c}x \overline{2} \\
\text { NoBolonia }\end{array}$ & DT & $\mathrm{t}(\mathrm{gl})$ & $\mathrm{P}$ \\
\hline $\begin{array}{c}\text { Hábitos de } \\
\text { Interés y } \\
\text { Concentración }\end{array}$ & 3,15 & ,749 & 3,28 &, 752 & $-3,78(1.290,62)$ &, $000 *$ \\
\hline $\begin{array}{c}\text { Superar las } \\
\text { Dificultades } \\
\text { Emocionales }\end{array}$ & 3,22 & ,641 & 3,27 &, 654 & $-1,66(1.256,12)$ & ,098 \\
\hline $\begin{array}{c}\text { Actividad y } \\
\text { Egoimplicación }\end{array}$ & 3,46 & ,631 & 3,56 &, 614 & $-3,41(1.315,31)$ &, 001 \\
\hline $\begin{array}{l}\text { Valoración } \\
\text { Logros }\end{array}$ & 3,82 & ,668 & 3,85 &, 623 & $-1,00(1.373,08)$ &, 317 \\
\hline $\begin{array}{l}\text { Sentido } \\
\text { Funcional }\end{array}$ & 3,26 &, 830 & 3,21 &, 863 & $1,31(1.251,14)$ & , 190 \\
\hline $\begin{array}{l}\text { Interconexión y } \\
\text { Ampliación }\end{array}$ & 3,61 & ,809 & 3,52 & ,794 & $2,44(1.305,73)$ &, 015 \\
\hline $\begin{array}{c}\text { Aproximación } \\
\text { Gradual }\end{array}$ & 3,33 & ,684 & 3,40 &, 641 & $-2,21(1.373,69)$ & ,027 \\
\hline $\begin{array}{l}\text { Anticipación } \\
\text { Facilitadora }\end{array}$ & 3,37 &, 825 & 3,46 &, 707 & $-2,72(1.490,88)$ & ,007 \\
\hline $\begin{array}{c}\text { Descarga ante } \\
\text { Tensión }\end{array}$ & 2,49 & ,792 & 2,71 &, 856 & $-5,76(1.209,09)$ &, $000 *$ \\
\hline $\begin{array}{l}\text { Descanso y } \\
\text { Variación }\end{array}$ & 3,67 &, 820 & 3,61 &, 785 & $1,42(1.344,16)$ & ,156 \\
\hline $\begin{array}{c}\text { Relajación y } \\
\text { Ánimo }\end{array}$ & 2,24 & ,685 & 2,38 &, 709 & $-4,34(1.252,98)$ &, $000 *$ \\
\hline
\end{tabular}

Tabla 7. Diferencias de medias t-Student para muestras independientes (HEME)

\section{DISCUSIÓN}

Tal y como se aprecia en los resultados obtenidos, podemos observar que los alumnos y alumnas pertenecientes al plan Bolonia hacen un uso más variado de estrategias cognitivas de aprendizaje, destacando entre ellas el aprendizaje mecánicosuperficial, el aprendizaje en voz alta, la utilización de la mnemotécnica, aprendizaje repetitivo y el aprendizaje colaborativo y oral. Sin embargo, y a pesar de utilizar un mayor número de estrategias, estos datos dejan vislumbrar cierta tendencia al aprendizaje poco elaborativo y en profundidad, decantándose por estrategias sencillas y de fácil aplicación, quizás centrándose más en la consecución del producto final que en la adquisición de conocimientos.

No obstante, y en coherencia con las recomendaciones de la EEES, se observa una preferencia por el aprendizaje colaborativo (G. López, et al., 2010, p. 154) por parte del alumnado Bolonia. Sin embargo, según las aportaciones de Gutiérrez-Braojos et al. (2009, p. 81), el éxito del aprendizaje colaborativo dependerá en gran medida de las 
convicciones previas del alumnado sobre el aprendizaje, es decir, si parten de una preferencia por el aprendizaje individualista o por el colaborativo/cooperativo.

Por el contrario, el alumnado que realizó sus estudios con el plan académico anterior al plan Bolonia, muestra un mayor uso de estrategias de esencialización y comprensión elaborada. Estos resultados indican que, con el plan antiguo, el alumnado llevaba a cabo su aprendizaje extrayendo y sintetizando las ideas principales, así como procurando una comprensión elaborada del contenido y adoptando un papel activo e interno de análisis y autoría en el estudio. Además, también muestran una mayor tendencia a la búsqueda de alternativas como estrategia de control del estudio, en comparación con el alumnado Bolonia. Informan de una mayor habilidad para aprender, afrontando las dificultades del estudio, buscando alternativas a los problemas o a la dificultad en la comprensión del contenido.

Autores y autoras como Trelles et al. (2018), Adame et al. (2013) o Monereo (2020) coinciden en que las estrategias más apropiadas para un mejor resultado académico están relacionadas con la elaboración y comprensión del contenido de estudio, tal y como se muestran en nuestro trabajo los estudiantes pertenecientes al plan de estudios anterior a Bolonia.

Aludiendo a los estilos de aprendizaje, se aprecia cómo los estudiantes Bolonia presentan estilos de aprendizaje con preferencia por el contenido teórico más que práctico, por los aspectos curiosos, anecdóticos y superficiales. Con ello se observa que no se ha logrado alcanzar uno de los objetivos principales del plan Bolonia, el cual se basaba en orientar al alumnado a un aprendizaje más práctico que teórico.

Por el contrario, el alumnado No Bolonia presenta un estilo de aprendizaje autorregulado, caracterizado por mostrarse insistentes, planificadores y autónomos en la revisión y el control del estudio. También manifiestan un estilo de aprendizaje inmerso, dado que se dejan llevar por la lectura durante el estudio, sin sentirse influenciados por las exigencias del profesor o del examen.

Por último, se observa que el alumnado No Bolonia utiliza una mayor variedad de estrategias motivacionales, destacando principalmente entre ellas, el interés y la concentración, la descarga ante tensión, la relajación y el ánimo. De estos datos podría deducirse que estos estudiantes han sido capaces o han visto la necesidad de desarrollar un abanico diverso de estrategias diferentes para automotivarse, lo que supondría un mayor conocimiento de sí mismos y de sus necesidades para conseguir mantener un nivel óptimo de estudio durante el proceso de aprendizaje.

En cuanto a las estrategias de control del estudio y los estilos de aprendizaje, observamos cómo el alumnado No Bolonia parece utilizar en mayor medida estrategias de control y estilos de aprendizaje autorregulado. Estás prácticas de estudio están apoyadas por numerosos autores y autoras como herramientas fundamentales para lograr el éxito académico (Núñez y González-Pumariega, 1996; Adame et al. 2013; Musso, 2016; Juárez et al. 2016; Teijeiro y Requies, 2018; Trelles et al. 2018; Navea y Varela, 2019; Hendrie y Bastacini, 2020).

Estos hallazgos nos obligan a reflexionar acerca de la efectividad de las recomendaciones implantadas por el plan Bolonia. A grandes rasgos podemos observar cómo los estudiantes de la Licenciatura de Psicología parecen mostrar estrategias de estudio y hábitos motivacionales más apropiados para alcanzar un adecuado rendimiento académico. Las aportaciones de M.I. López et al. (2016) coinciden con nuestros resultados, ya que afirman encontrar datos similares, demostrando un mejor 
rendimiento académico de los estudiantes de Licenciatura en comparación con los estudiantes de Grado.

En líneas de trabajo similares, pero desde la perspectiva del profesorado universitario, Quevedo-Blasco y Buela-Casal (2017) señalan en su estudio que los docentes manifiestan no sentirse cualificados para poder adaptarse a los criterios establecidos por el EEES, por lo que consideran imprescindible una mejora significativa en esta transición. Sin embargo, estos hallazgos contradicen lo presentado por un informe sobre el aprendizaje y la enseñanza en el área de Educación Superior Europea. En esta publicación se destaca como uno de los objetivos estratégicos en el marco educativo europeo el desarrollo del personal académico, mostrando numerosos datos e indicadores que, salvo algunas diferencias entre países, argumentan positivamente la consecución a grandes rasgos de este objetivo a través de la disposición de recursos, materiales, asesoramiento al docente $\mathrm{y} / \mathrm{o}$ colaboraciones entre facultades $\mathrm{y}$ departamentos (Gaebel et al., 2018). Estos hechos podrían indicar cierta falta de comunicación entre los organismos europeos y las vivencias reales del personal académico, afectando por ende al aprendizaje del alumnado.

De lo expuesto cabría preguntarse, ¿por qué el alumnado de Psicología de la ULL no ha conseguido mejorar el uso de estrategias, de estilos de aprendizaje y de control del estudio, así como de estrategias motivacionales con las medidas del plan Bolonia? Desde nuestra perspectiva, consideramos que quizás las exigencias para homogeneizar los planes de estudio de los países europeos planteadas por el EEES se enfocan en mayor medida en aspectos estructurales y de unificación de criterios burocráticos, más que en desarrollar y proponer medidas detalladas y concretas sobre la metodología didáctica para aplicar con el plan Bolonia.

Estas reflexiones coinciden con las aportadas por Bolívar (2007, p. 90), quien concluye en su estudio que "la reforma exigida por el plan Bolonia parece limitarse a una reforma estructural más, sin lograr la mejora del proceso de enseñanzaaprendizaje". Por su parte M.I. López et al. (2016), consideran que este desfase en el rendimiento de los graduados podría deberse en parte, a la falta de recursos tanto humanos como materiales y a la infravaloración de la práctica docente, aspectos que el plan Bolonia no ha tenido en cuenta lo suficiente. Por el contrario, otra investigación realizada con estudiantes del Grado de Trabajo Social, muestra que el alumnado se encuentra satisfecho con las competencias profesionales específicas aportadas por dicho título amparado bajo el EEES (Carrión-Martínez et al., 2018).

En coherencia con nuestro trabajo, destacamos las conclusiones presentadas por un estudio realizado con alumnado de Psicología. En esta investigación informan de la baja satisfacción por parte del profesorado universitario ante la implantación del Plan Bolonia en las universidades españolas (Ariza et al., 2014; Quevedo-Blasco, Ariza y Buela-Casal, 2015). Sin embargo, en relación con el objeto de su estudio, sus resultados mostraron un considerable aumento en la realización de tesis doctorales en Psicología después de la adaptación al EEES.

Por otro lado, y en línea con nuestros hallazgos, según un trabajo realizado por Quevedo-Blasco y Buela-Casal (2019), la mitad del profesorado participante en su investigación cuestionó los efectos positivos de la implantación del plan Bolonia. Otros estudios, sostienen que no se ha observado una mejora en la adquisición de conocimientos por parte de estos estudiantes (Ramiro-Sánchez et al. 2016), así como 
una menor independencia y autonomía del alumnado respecto a su aprendizaje (BarbaMartín et al., 2020).

\section{CONCLUSIONES}

En congruencia con nuestros resultados, podríamos establecer la existencia de dos perfiles claramente diferenciados en referencia a la forma de llevar a cabo el aprendizaje. Por un lado, un alumno "obrero", el cual lleva a cabo su aprendizaje con la expectativa de un feedback inmediato del profesorado, siendo más ejecutivo, atomizado y centrado en la tarea, a través de estrategias de aprendizaje básicas (alumno Bolonia). Y, por otro lado, un estudiante "pensante", más autónomo, más orientado a la toma de decisiones, a la búsqueda de alternativas y más elaborativo (alumno No Bolonia). No obstante, el mayor uso de estrategias motivacionales podría indicar una mayor necesidad de automotivarse durante su aprendizaje.

Ante estas conclusiones, consideramos necesaria una revisión inmediata de las directrices que ha de seguir la comunidad educativa para alcanzar los objetivos propuestos por la EEES (G. López et al., 2010, p. 154) y quizás proponer un plan de seguimiento y evaluación periódica de la metodología aplicada al proceso de enseñanzaaprendizaje universitario. Desde otro punto de vista, Chen et al. (2021) plantean que la atención para la mejora del aprendizaje del alumnado debe ir más allá de la transmisión del conocimiento y reorientarse más hacia cómo se transmiten los contenidos, utilizando las TICs como herramienta principal y manteniendo a la comunidad educativa a la vanguardia de los avances tecnológicos. Estas tendencias se han desarrollado en mayor medida por el efecto de la pandemia del Covid-19 en la educación. Se ha hecho patente que las nuevas generaciones, sobre todo de estudiantes universitarios, requieren un cambio en la gestión de los contenidos y una enseñanza-aprendizaje mixta entre lo digital y lo presencial (Muñoz, 2021). Consideramos interesante tener en cuenta estas actuales perspectivas para próximas líneas de estudio relacionadas con el ámbito educativo universitario.

Indudablemente, este estudio nos ha mostrado las actuales deficiencias del plan Bolonia en los estudiantes del Grado de Psicología, respondiendo así a nuestra hipótesis de trabajo. Por ello consideramos necesaria la realización de estudios similares para contrastar nuestros resultados con otras especialidades de mayor contenido práctico.

\section{BIBLIOGRAFÍA}

Adame, M.T., Aguilar, E.M., Casero, A., Colom, J., De la Iglesia, B., Grases, G., Luca de Tena, C., Pérez, J.A., Rigo, E., Rodríguez, R.I., Rosselló, M.R. y Sureda, I. (2013). Estrategias de estudio y trabajo en estudiantes universitarios de la UIB. Institut de Recerca i Innovació Educativa, 7, 1-178. https://doi.org/10.3306/IRIE.INFORME.RECERCA.N7.2013

Amber, D. y Martínez-Valdivia, E. (2018). La formación en Educación Superior. Retos y propuestas en docencia universitaria. Profesorado, Revista de Currículum y Formación del Profesorado, 22(3), 1-8. https://doi.org/10.30827/profesorado.v22i3.7987

Ariza, T., Quevedo-Blasco, R. y Buela-Casal, G. (2014). Satisfaction of Social and Legal Sciences teachers with the introduction of the European Higher Education Area. The European Journal of Psychology Applied to Legal Context, 6(1), 9-16. https://doi.org/10.5093/ejpalc2014a2 
Barba-Martín, R., Hernando-Garijo, A., Hortigüela-Alcalá, D. y González-Calvo, G. (2020). Tras casi una década de Bolonia: ¿realmente hemos mejorado la calidad de la enseñanza? Espiral. Cuadernos del profesorado, 13(27), 97-108.

Bolívar, A. (2007). La planificación por competencias en la reforma de Bolonia de la educación superior: un análisis crítico. ETD - Educação Temática Digital. Recuperado de: https://nbn-resolving.org/urn:nbn:de:0168-ssoar-73427

Brosig, M.E., Araiza, M.J. y Niño, C. (2021). Estilos de Aprendizaje en una Escuela de Negocios, Puntuaciones Promedio, Diferencias y Directrices en el Aprendizaje. Revista Daena (International Journal of Good Conscience), 16(2), 1-12.

Carneiro-Barrera, A., Ruiz-Herrera, N. y Díaz-Román, A. (2019). Tesis doctorales en Psicología tras la adaptación al Espacio Europeo de Educación Superior. Revista de Investigación en Educación, 17(1), 32-43. Recuperado de: http://webs.uvigo.es/reined/

Carrión-Martínez, J.J., Fernández-Martínez, M.M., Pérez-Fuentes, M.C. y Gázquez-Linares, J.J. (2018). Competencias específicas en la educación superior en trabajo social en el marco del espacio europeo de educación superior: la percepción de los futuros profesionales en el contexto español. Revista europea de trabajo social, 23 (1), 1-13. https://doi.org/10.1080/13691457.2018.1460717

Cazorla, M.C. (2011). Una aproximación a los aspectos positivos y negativos derivados de la puesta en marcha del Plan Bolonia en la Universidad Española. REJIE: Revista Jurídica de Investigación e Innovación Educativa. Recuperado de: http://www.eumed.net/rev/rejie

Chen, X., Zou, D., Xie, H. y Wang, F.L. (2021). Past, present, and future of smart learning: a topic-based bibliometric analysis. International Journal of Educational Technology in Higher Education, 18(1), 1-29. https://doi.org/10.1186/s41239-020-00239-6

Corominas, E. (2001). Competencias genéricas en la formación universitaria. Revista de educación. Recuperado de: http://hdl.handle.net/11162/75927

Correa, C. (2019). Estrategias didácticas para la formación en investigación social en América latina. La construcción de marcos teóricos y pensamiento epistémico. Revista de Educación de la Universidad de Granada, 26, 143-161. http://doi.org/10.30827//reugra.v26i0.123

Díez, E.J. (2009). El capitalismo académico y el plan Bolonia. Eikasia: Revista de Filosofía, año IV. Recuperado de: http://hdl.handle.net/10612/11807

Dirección General de Educación y Cultura (2002). Las competencias clave. Un concepto en expansión dentro de la educación general obligatoria. Eurydice.

Esguerra, G. y Guerrero, P. (2010). Estilos de aprendizaje y rendimiento académico en estudiantes de Psicología Diversitas. Perspectivas En Psicología, 6(1), 97-109.

European Commission/EACEA/Eurydice (2020). The European Higher Education Area in 2020: Bologna Process Implementation Report. Publications Office of the European Union.

Gaebel, M., Zhang, T., Bunescu, L. y Stoeber, H. (2018). Trends 2018. Learning and teaching in the European higher education area. European University Association asbl.

Gutierrez-Braojos, C., Salmerón, P. y Muñoz de Escalona, M. (2009). El aprendizaje autorregulado y las concepciones de los estudiantes en el aprendizaje cooperativo. Revista de Educación de la Universidad de Granada, 22(2), 73-82.

Guzmán-Valenzuela, C., Gómez-González, C., Rojas-Murphy, A. y Lorca-Vyhmeister, A. (2021). Learning analytics in higher education: a preponderance of analytics but very little learning? International Journal of Educational Technology in Higher Education, 18(1), 119. https://doi.org/10.1186/s41239-021-00258- $x$

Hendrie, K.N. y Bastacini, M. (2020). Autorregulación en estudiantes universitarios: Estrategias de aprendizaje, motivación y emociones. Revista Educación, 44(1), 327-344. https://doi.org/10.15517/revedu.v44i1.37713

Hernández, P. y García, L.A. (1991). Psicología y enseñanza del estudio: Teorías y técnicas para potenciar las habilidades intelectuales. Pirámide. 
Jefatura del Estado. Gobierno de España. (2001, 24 de diciembre). Ley Orgánica 6/2001, de 21 de diciembre, de Universidades. Boletín Oficial del Estado, núm. 307. https://www.boe.es/buscar/pdf/2001/BOE-A-2001-24515-consolidado.pdf

Jefatura del Estado. Gobierno de España. (2007, 13 de abril). Ley Orgánica 4/2007, de 12 de abril, por la que se modifica la Ley Orgánica 6/2001, de 21 de diciembre, de Universidades. Boletín Oficial del Estado, núm. 89. https://www.boe.es/eli/es/lo/2007/04/12/4

Juárez, C., Rodríguez, G., Escoto, M. y Luna, E. (2016). Relación de los estilos y estrategias de aprendizaje con el rendimiento académico en estudiantes universitarios. Revista de Estilos de Aprendizaje, 9(17). Recuperado de: http://revistaestilosdeaprendizaje.com/article/view/1054

López, G., de Oña, R., Garach, L., Calvo, F.J. y de Oña, J. (2010). El nuevo rol del alumno como grupo profesor como herramienta para alcanzar algunos de los objetivos perseguidos en Bolonia. En Actas de las I Jornadas sobre Innovación Docente y Adaptación al EEES en las Titulaciones Técnicas (pp. 153-158). Godel Impresiones Digitales, SL.

López, J.A., Lorenzo, M.E. y Trujillo, J.M. (2009). Estudio sobre el proceso de enseñanzaaprendizaje en las universidades populares españolas. Una visión desde los propios implicados. Revista de Educación de la Universidad de Granada, 22(2), 121-142.

López, M.I., Palací, D.G. y Palací, J. (2016). Disminución del rendimiento académico con el Plan Bolonia respecto al plan anterior en España. Revista Complutense de Educación, 27(2), 633-651. https://doi.org/10.5209/rev_RCED.2016.v27.n2.46915

Martín, E., García, L.A., Torbay, Á. y Rodríguez, T. (2007). Estructura factorial y fiabilidad de un cuestionario de estrategias de aprendizaje en universitarios: CEA-U. Anales de Psicología/Annals of Psychology, 23(1), 1-6.

Martín, E., García, L.A., Torbay, Á. y Rodríguez, T. (2008). Estrategias de aprendizaje y rendimiento académico en estudiantes universitarios. International Journal of Psychology and Psychological Therapy, 8(3), 401-412. Recuperado de: http://www.redalyc.org/articulo.oa?id=56080312

Ministerio de Educación y Ciencia. Gobierno de España. (2007, 30 de octubre). Real Decreto 1393/2007, de 29 de octubre, por el que se establece la ordenación de las enseñanzas universitarias oficiales. Boletín Oficial del Estado núm. 260. https://www.boe.es/eli/es/rd/2007/10/29/1393/con

Ministerio de Universidades. Gobierno de España. (2021, 29 de septiembre). Real Decreto $822 / 2021$, de 28 de septiembre, por el que se establece la organización de las enseñanzas universitarias y del procedimiento de aseguramiento de su calidad. Boletín Oficial del Estado núm. 233. https://www.boe.es/eli/es/rd/2021/09/28/822/con

Monereo, C.F. (2020). Enseñar y aprender en la educación superior. En M. Turull (Coord.), Manual de docencia universitaria (pp. 75-98). Ediciones Octaedro.

Muñoz, I. (2021). Nuevos modelos de docencia, desde la declaración de Bolonia a la era de la COVID. Miscelánea Comillas. Revista de Ciencias Humanas y Sociales, 79(154), 225-253. https://doi.org/10.14422/mis.v79.i154.y2021.007

Musso, M.F. (2016). Understanding the underpinnings of academic performance: Students, asks and performance: A structural equations (SEM) approach to model the effect of cognitive and SRL factors in academic performance. (Doctoral thesis). KU Leuven: Bélgica.

Navea, A. y Varela, I. (2019). Variables motivacionales y cognitivas predictivas del rendimiento en estudiantes universitarios de ciencias de la salud. Educación Médica Superior, 33(1).

Núñez, J.C. y González-Pumariega, S. (1996). Procesos motivacionales y aprendizaje. Psicología de la instrucción, 2, 33-64.

Perrenoud, P. (2001). Porquê construir competências a partir da escola? Desenvolvimento da autonomia e luta contra as desigualdades. Ediçoes ASA.

Quevedo-Blasco, R., Ariza, T. y Buela-Casal, G. (2015). Evaluación de la satisfacción del profesorado de Ciencias con la adaptación al Espacio Europeo de Educación Superior. Educación XX1, 18(1), 45-70. https://doi.org/10.5944/educXX1.18.1.12311 
Quevedo-Blasco, R. y Buela-Casal, G. (2017). Influencia de la implantación del espacio europeo de educación superior en el profesorado universitario de ingeniería y arquitectura. Dyna, 92(3), 333-338. https://doi.org/10.6036/8045

Quevedo-Blasco, R. y Buela-Casal, G. (2019). Satisfacción del profesorado en España con el Espacio Europeo de Educación Superior: Artes y Humanidades. Universitas Psychologica, 18(5). Recuperado de:

https://link.gale.com/apps/doc/A628846876/AONE?u=anon 7e6acbc6\&sid=googleScholar \&xid $=c 8936 b 75$

Ramiro-Sánchez, T., Bermúdez, M.P. y Buela-Casal, G. (2016). Titulación de Psicología en el EEES: comparación entre licenciados y graduados españoles. Revista de Psicodidáctica, 21(1), 175- 189. https://doi.org/10.1387/RevPsicodidact.13923

Rué, J. (2007). Enseñar en la Universidad: El EEES como reto para la Educación Superior (Vol. 16). Narcea Ediciones.

Soflano, M., Connolli, T.M. y Haney, T. (2015). Learning style analysis in adaptive GBL application to teach SQL. Computers \& Education, 86, 105-119. https://doi. org/10.1016/j.compedu.2015.02.009

Teijeiro, M.E. y Requies, I. (2018). Estrategias de aprendizaje y competencias del grado de maestro/a en Educación Primaria. Profesorado, Revista de Currículum y Formación del Profesorado, 22(3), 75-95. https://doi.org/10.30827/profesorado.v22i3.7993

Trelles, H.J., Alvarado, H.P. y Montánchez, M.L. (2018). Estrategias y estilos de aprendizaje y su relación con el rendimiento académico en estudiantes universitarios de Psicología Educativa. Killkana Social, 2(2), 9-16. https://doi.org/10.26871/killkana_social.v2i2.292

Zimmerman, B.J. (2000). Attaining self-regulation: A social cognitive perspective. En M. Boekaerts, P. Pintrich, y M. Zeidner (Eds.), Handbook of self-regulation (pp. 13-39). Academic Press.

Zimmerman, B.J. y Dibenedetto, M.K. (2008). Mastery learning and assessment: Implications for students and teachers in an era of high-stakes testing. Psychology in the Schools, 45(3), 206-216. https://doi.org/10.1002/pits.20291

Zimmerman, B.J. y Martinez-Pons, M. (1988). Construct validation of a strategy model of student self-regulated learning. Journal of Educational Psychology, 80, 284-290. 